\title{
Unconjugated Bile Acids Modulate Adult and Neonatal Neutrophil Chemotaxis Induced In Vitro by $N$-Formyl-Met-Leu-Phe-Peptide
}

\author{
PASQUALE SANTORO, FRANCESCO RAIMONDI, SIMONA ANNUNZIATA, \\ ROBERTO PALUDETTO, TIZIANA ANNELLA, AND FILIPPO CICCIMARRA \\ Division of Neonatology, Department of Pediatrics, "Federico II" University, 80131 Naples, Italy [P.S., \\ F.R., S.A., R.P., T.A., F.C.]; Division of Neonatology, University of Maryland, Baltimore, MD, U.S.A. \\ [F.R.]
}

\begin{abstract}
In this study, we have investigated the effect of hydrophobic and hydrophilic unconjugated bile acids (UBAs) - ursodeoxycholic acid (UDCA), chenodeoxycholic acid (CDCA), lithocholic acid, and colic acid-on chemotaxis in adult and neonatal human polymorphonuclear leukocytes (PMNs). The trypan blue exclusion dye test was preliminarily performed to determine the toxicity of the studied UBAs on PMNs. N-formyl-methionylleucyl-phenylalanine $(100 \mathrm{nM})$ was used as a chemoattractant. Chemotaxis $\left(1 \times 10^{6}\right.$ cells $\left./ \mathrm{mL}\right)$ was analyzed in the presence or absence of UBAs $(10 \mu \mathrm{M})$ by blind well chambers. The antioxidants vitamin $\mathrm{E}$ and vitamin $\mathrm{C}$ were tested for their ability to reduce the inhibitory effect of UBAs. We found that only CDCA was able to induce damage in PMNs in the range of $1-40 \mu \mathrm{M}$. Both CDCA and UDCA were able to inhibit chemotaxis in PMNs, whereas lithocholic acid and colic acid were ineffective. The inhibitory effect was reversible inasmuch as PMNs incubated with either CDCA or UDCA and subsequently washed showed normal chemotaxis. Concomitant incubation of PMNs with UBAs and vitamins $\mathrm{C}$ or E reversed the inhibition. We did
\end{abstract}

\section{ABSTRACT}

not find substantial differences between PMNs from adults or newborns. In conclusion, CDCA and UDCA are able to reduce, in a specific and reversible fashion, both adult and newborn neutrophil chemotaxis. As concomitant incubation of UBAs and electron scavengers restores PMN chemotaxis to control values, we conclude that free radicals may be involved in the mechanism of inhibition. We speculate that this defect may contribute to the impaired host response described in cholestatic patient. (Pediatr Res 51: 392-396, 2002)
Abbreviations
PMN, polymorphonuclear leukocytes
UBA, unconjugated bile acid
UDCA, ursodeoxycholic acid
CDCA, chenodeoxycholic acid
LTCA, lithocholic acid
CA, colic acid
fMLP, N-formyl-methionyl-leucyl-phenylalanine

The high incidence of postsurgical septic complications in patients with obstructive jaundice (1-3) and the wide use of UDCA in human therapy (4-6) have prompted previous investigators to study the effect of both bile as a whole and UBAs on PMN function. However, conflicting results have been obtained. In surgical models of cholestasis in the rat, both depressed $(7,8)$ and enhanced (9) PMN functions have been described. Recently, D'Odorico et al. (10) reported no altered production of reactive oxygen metabolites in neutrophils from patient with chronic cholestatic liver disease. In contrast, others (11) described increased hydrogen peroxide and superoxide anion generation from patients with obstructive jaundice. These differences may be explained by different experimental conditions and/or by the experimental mod-

Received May 8, 2001; accepted November 7, 2001.

Correspondence: Pasquale Santoro, Università Federico II, Dipartimento di Pediatria, Via Sergio Pansini, 5-80131 Napoli, Italy; e-mail: pasantor@unina.it

F.R. was recipient of a "Nicholas Green Foundation" grant. els used, e.g. the profound differences that exist between bile acid metabolism in rats and humans (12). Another cause of bias may be the peculiar ability of PMNs to easily undergo activation or to be primed, conditions that may occur during surgical manipulation of rats.

Only one study, to our knowledge, has previously investigated the effect of bile as a whole on PMN chemotaxis (9), whereas Chen et al. (13) recently described the ability of CDCA to act as an antagonist of the formyl-peptide receptor in human monocytes and in transfected cells. However, these authors used bile acids at high concentrations and did not provide any information about their toxicity on human neutrophils.

The high incidence of hepatobiliary disease in newborns that have defective PMN function has been observed (14-16), however, the effect of UBAs on the natural immune systems of neonates has not been investigated to date. 
The aim of our work was to study the effect of individual UBAs on both adult and newborn human PMN chemotaxis in an in vitro model. We report the effect of UBAs differing in their hydrophilic/hydrophobic properties: from the relatively hydrophilic $\mathrm{CA}(3 \alpha, 7 \alpha, 12 \alpha$-trihydroxy- $5 \beta$-cholan-24-oic acid), to $\operatorname{CDCA}(3 \alpha, 7 \alpha$-dihydroxy-5 $\beta$-cholan-24-oic acid) and its $7 \beta$-epimer UDCA (widely used in bile acid therapy), to the most hydrophobic, LTCA ( $3 \alpha$-hydroxy, $5 \beta$-cholan-24-oic acid).

\section{MATERIAL AND METHODS}

Chemicals. All culture media were purchased as sterile and endotoxin tested solutions from Sigma Chemical (St. Louis, MO, U.S.A.).

UDCA, CDCA, LTCA, CA, fMLP, vitamin C, vitamin E, Ficoll-Hypaque, and Dextran were also obtained from Sigma Chemical. Other products were of analytical reagent grade. Bile acids $(500 \mathrm{mM})$ were dissolved in DMSO and stored at $4^{\circ} \mathrm{C}$ for no longer than a week. Working solutions were prepared just before use in RPMI media.

Because of the low solubility of vitamin $\mathrm{E}$ in water, buffers, DMSO, and similar partially polar solvents, and because of the ability of ethanol to interfere with PMN functions (17), we dissolved the vitamins in diethyl ether. The exact amount of diethyl ether dissolved vitamin $\mathrm{E}$ required for each experiment was pipetted into the appropriate vial. The solvent was evaporated in a vacuum overnight under agitation. Then, one half of the final volume of tissue culture media was added and the vials were vortexed extensively. Lastly, we added cells or UBAs to give the final concentrations mentioned in the text. Control samples were prepared accordingly.

Sampling of blood. The local ethical committee approved this study. Mothers gave consent for cord blood collection before delivery. Similarly, adult volunteers gave their consent for blood drawing.

Heparin at a concentration of $20 \mathrm{U} / \mathrm{mL}$ was used as anticoagulant. Venous blood was collected from the placental vessels of full-term infants who were delivered vaginally. Newborns were the product of uncomplicated pregnancies and deliveries; neither general nor local anesthesia was administered to the mothers. No perinatal complications occurred in any of the babies studied, and all had an Apgar score $\geq 8$ at 1 and $5 \mathrm{~min}$. Blood from healthy adult donors was drawn from the forearm vein and studied simultaneously with cord blood samples.

PMN purification. Immediately after blood collection, blood was layered over Ficoll-Hypaque (density $=1.077$ ) and centrifuged at $400 \times g$ for $30 \mathrm{~min}$ to remove mononuclear cells.

Erythrocytes were separated by $2 \%$ dextran $\left(M_{\mathrm{r}} 480,000\right)$ sedimentation for $30 \mathrm{~min}$ at room temperature. Residual erythrocytes were removed by hypotonic lysis with cold $0.2 \% \mathrm{NaCl}$ for exactly $30 \mathrm{~s}$. Adding an equal volume of cold $1.6 \% \mathrm{NaCl}$ restored isotonicity.

Cells were washed twice and suspended in RPMI containing $5 \%$ FCS. PMNs obtained by this technique were $\geq 97 \%$ pure and $\geq 99 \%$ viable.

Trypan blue exclusion dye test. Trypan blue exclusion dye test was used to determine the toxicity of UBAs. Cells were incubated for $45 \min \left(37^{\circ} \mathrm{C}, 5 \% \mathrm{CO}_{2}, 95 \%\right.$ relative humidity $)$ at various UBA concentrations in the presence of $0.2 \%$ trypan blue. Stained PMNs were counted at a magnification of $\times 400$.

Chemotaxis. fMLP (100 nM) was used as a chemoattractant. This was the minimal concentration required to stimulate the maximal chemotactic response from human neutrophils (not shown). Cells $\left(1 \times 10^{6} / \mathrm{mL}\right)$ were loaded in the upper well of blind chambers $(200 \mu \mathrm{L}$, NeuroProbe, Gaithersburg, MD, U.S.A.) equipped with $3-\mu \mathrm{m}$ polycarbonate filters. DMSOdissolved UBA was added to PMN suspensions at a final concentration of $10 \mu \mathrm{M}$ (DMSO $<0.01 \%$ ). After the appropriate incubation period $\left(30 \mathrm{~min}\right.$ at $37^{\circ} \mathrm{C}, 5 \% \mathrm{CO}_{2}, 95 \%$ relative humidity) filters were removed and fixed by immersion in methanol. Cells were stained with $10 \%$ Giemsa solution. The cells that migrated through the pores were counted at 1000 -fold magnification from four randomly selected fields. PMNs that dropped off the membrane were recovered from the lower chamber, counted, and added to the total number of migrated cells.

Reversibility of the inhibition induced by CDCA and UDCA. The appropriate bile acid was added to the PMN suspension (in these experiments, the cell concentration was 2 $\times 10^{6} / \mathrm{mL}$ to compensate for the loss of cells that occurs during the following centrifugation steps) and incubated for $20 \mathrm{~min}$ at $37^{\circ} \mathrm{C}$. Cells were then washed three times with the culture media and suspended to the initial volume. Chemotaxis was performed as already described.

Control tubes were prepared as follows: PMN suspensions $\left(2 \times 10^{6} / \mathrm{mL}\right)$ were incubated with the solvent alone for $20 \mathrm{~min}$ at $37^{\circ} \mathrm{C}$, washed three times with the culture media, and then the appropriate bile acid (or the solvent alone) was added. Chemotaxis was performed as above.

We also tested the ability of the antioxidant vitamins $\mathrm{E}$ and $\mathrm{C}$ to revert the inhibitory effect of CDCA or UDCA as an indirect evidence of involvement of free radicals in the mechanism of chemotaxis inhibition $(18,19)$. In these experiments, the vitamins were added concomitantly with the UBAs, without any preincubation.

Statistical analysis. The significance of differences between the groups was tested by the one-way ANOVA. We used Tukey's post hoc test to compare pairs of group means. Results are reported as mean $\pm \mathrm{SD}$.

\section{RESULTS}

Cell viability. Cell viability was studied at UBA concentrations ranging from 1 to $40 \mu \mathrm{M}$. As shown in Figure 1, only CDCA demonstrated a clear dose-response cytotoxicity for human PMNs. In the presence of $5 \mu \mathrm{M}$ CDCA, trypan bluepositive cells increased from $0.53 \pm 0.28 \%$ (control) to $5.7 \pm$ $1.2 \%$ for the adult PMNs and from $0.8 \pm 0.91 \%$ (control) to $4.8 \pm 0.65 \%$ for the cord blood PMNs (mean $\pm \mathrm{SD} ; p<0.01$, $n=4)$. At the maximal concentration tested $(40 \mu \mathrm{M})$, CDCAinduced cell death increased up to $21.98 \pm 0.75 \%$ and $24 \pm$ $3.00 \%$ in PMNs from both adults and neonates, respectively. The other UBAs did not significantly affect cell viability except for a slight but not significant ( $p>0.05, n=4)$ increment of trypan blue-positive cells observed in the presence of $40 \mu \mathrm{M}$ 


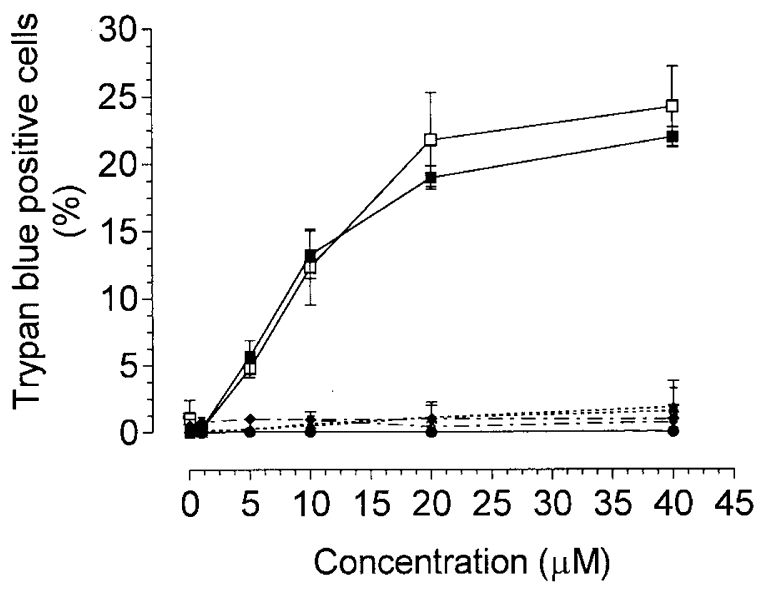

Figure 1. Cell damage induced by bile acids in human PMNs. Only CDCA was able to induce significant cell injury and consequent staining of PMNs by the trypan blue exclusion dye test. The presence of the other bile acids did not significantly affect cell viability. Experiments performed with adult PMNs: filled squares $=\mathrm{CDCA} ;$ filled triangles $=\mathrm{UDCA} ;$ filled circles $=\mathrm{LTCA}$; filled diamonds $=\mathrm{CA}$. Experiments performed with neonatal PMNs: open squares $=\mathrm{CDCA} ;$ open triangles $=\mathrm{UDCA} ;$ open circles $=\mathrm{LTCA} ;$ open diamonds $=\mathrm{CA}$.

UDCA. We did not find significant differences between UBA toxicity in adult or cord blood PMNs.

Inhibition of fMLP-induced PMN locomotion. Both CDCA and UDCA $(10 \mu \mathrm{M})$ were able to inhibit significantly fMLPinduced chemotaxis $(p<0.01, n=6)$. The effect was similar in adult and neonatal samples. Neither LTCA nor CA inhibited cell locomotion significantly ( $p>0.05, n=6$ ). Results are summarized in Figure 2.

As reported, as the concentration of CDCA and UDCA increases, the chemotaxis, expressed as a percentage of the maximal response in the absence of inhibitor, decreases. Concentrations of 20 and $40 \mu \mathrm{M}$ resulted in the inhibition of the random PMN motility.

It should be pointed out that at the concentrations of 1 and $5 \mu \mathrm{M}$, UDCA, despite the lower toxicity, was more effective than CDCA (33.22 $\pm 2.26 \%$ versus $87.71 \pm 0.90 \%$; $30.29 \pm$ $2.72 \%$ versus $46.01 \pm 2.47 \%$ for the adults and $34.67 \pm$ $9.484 \%$ versus $84.79 \pm 5.643 \% ; 36.15 \pm 4.186 \%$ versus 45.54 $\pm 3.339 \%$ in neonatal PMNs, respectively; $p<0.001, n=6$ ).

Chemotaxis inhibition is reported as a percentage of the maximal response against a control chemotaxis. This allows a direct comparison between adult and neonatal samples. However, as already reported, neonatal PMNs have a natural decreased motility (14).

Reversibility of chemotaxis inhibition. Human PMNs incubated with either CDCA or UDCA and subsequently washed showed a chemotactic response not significantly different from control fMLP-induced chemotaxis (for CDCA and UDCA: $110.70 \pm 18.04 \%$ and $104.30 \pm 5.32 \%$ for the adult; and 105 $\pm 16.4 \%$ and $110.50 \pm 17.0 \%$ for neonatal PMNs, respectively). These results were from six separate experiments.

Effect of vitamin $C$ and vitamin $E$ on bile acid-induced chemotaxis inhibition. Both vitamins were capable of wholly restoring cell locomotion in a dose-dependent manner. In

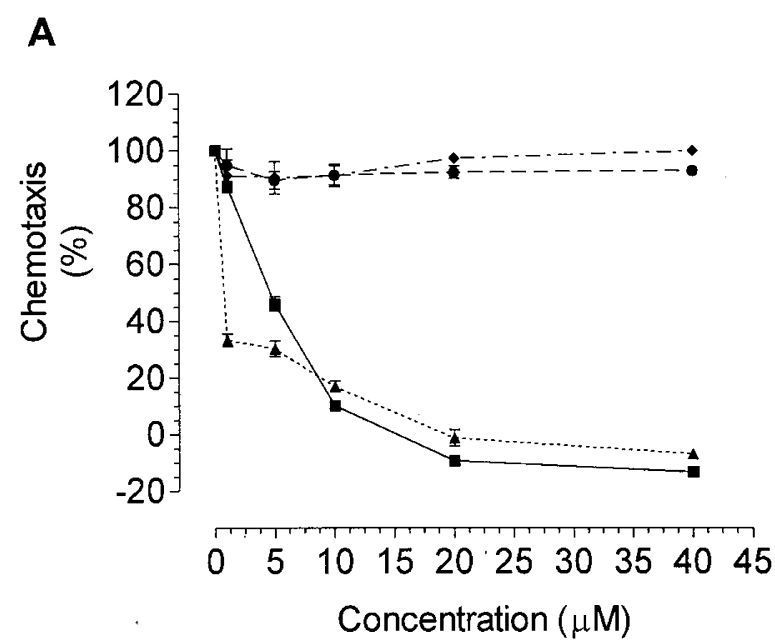

B

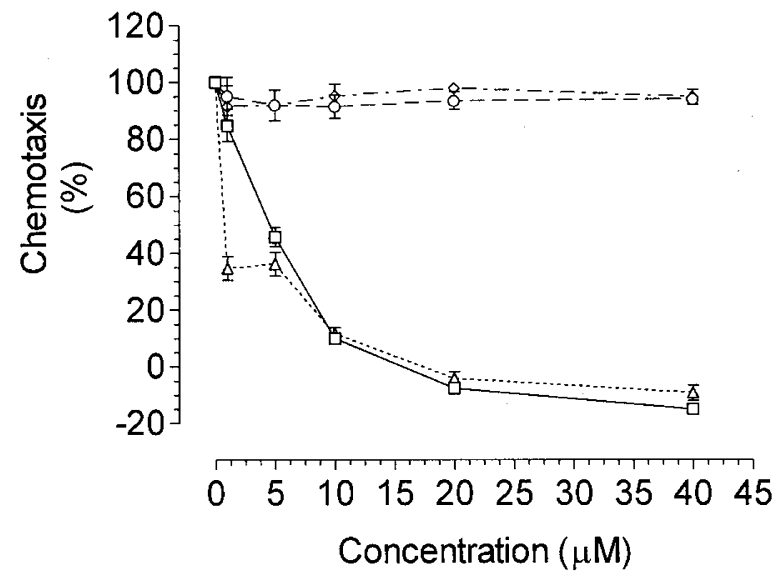

Figure 2. Effect of bile acids on fMLP-induced chemotaxis. Both CDCA and UDCA were able to reduce significantly the fMLP-induced chemotaxis in both adult (panel $A$ ) and neonatal samples (panel $B$ ). Similar results were obtained from adult and neonatal PMNs. Results were normalized assuming control $=$ 0 and fMLP-stimulated chemotaxis $=100$. Experiments performed with adult PMNs: filled squares $=\mathrm{CDCA} ;$ filled triangles $=\mathrm{UDCA}$; filled circles $=$ LTCA; filled diamonds $=$ CA. Experiments performed with neonatal PMNs: open squares $=\mathrm{CDCA} ;$ open triangles $=\mathrm{UDCA} ;$ open circles $=\mathrm{LTCA} ;$ open diamonds $=\mathrm{CA}$.

particular, $100 \mu \mathrm{M}$ vitamin $\mathrm{C}$ could reverse the inhibitory effect of UBAs on fMLP-induced chemotaxis (Fig. 3).

Vitamin E had similar effects on human PMNs, but millimolar concentrations were needed to restore the chemotactic response (Fig. 4). This may the result of the low solubility of vitamin $\mathrm{E}$ in aqueous solutions. For both vitamins, similar results were obtained in adult and neonatal PMNs.

\section{DISCUSSION}

In this study, we have tested individual UBAs in an in vitro system to determine their ability to impair PMN chemotaxis. The concentrations used are relevant to most clinical situations. In fact, normal serum concentrations of each specific UBA range between 0.2 and $1 \mu \mathrm{M}(20)$. However, they may increase up to $150 \mu \mathrm{M}$ in chronic cholestatic disease (21). Moreover, 


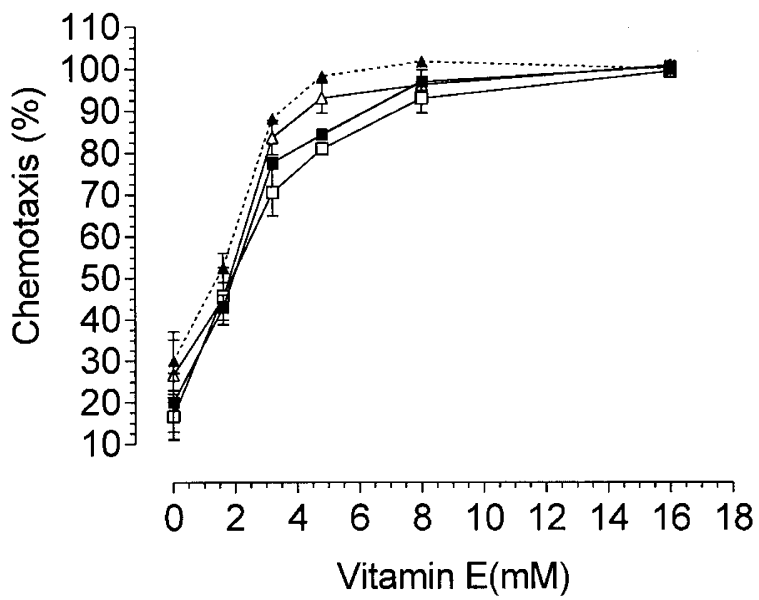

Figure 3. Dose-dependent effect of vitamin $\mathrm{C}$ on fMLP-induced chemotaxis inhibited by either CDCA or UDCA $(10 \mu \mathrm{M})$. Vitamin $\mathrm{C}$ is capable of reversing the inhibitory effect of bile acids on fMLP-induced chemotaxis at a concentration of $100 \mu \mathrm{M}$ in both adult and neonatal samples. Results were from three separate experiments reported as normalized, assuming control $=0$ and the fMLP-stimulated chemotaxis $=100$. Experiments performed with adult PMNs: filled squares $=$ CDCA; filled triangles $=$ UDCA. Experiments performed with neonatal PMNs: open squares $=\mathrm{CDCA}$; open triangles $=$ UDCA.

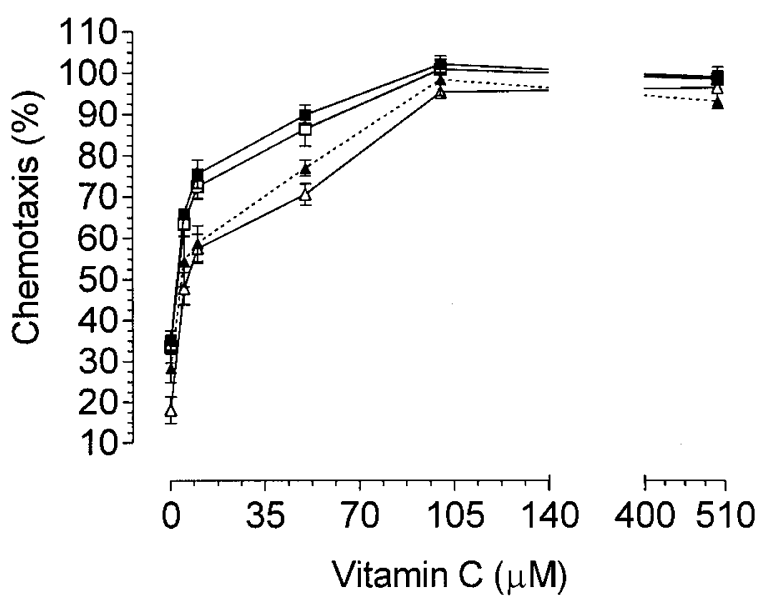

Figure 4. Dose-dependent effect of vitamin E on fMLP-induced chemotaxis inhibited by either CDCA or UDCA $(10 \mu \mathrm{M})$. Vitamin $\mathrm{E}$ is capable of reversing the inhibitory effect of bile acids on fMLP-induced chemotaxis at a concentration of $8 \mu \mathrm{M}$. Similar results were obtained from adult and neonatal samples. Results were from three separate experiments reported as normalized, assuming control $=0$ and the fMLP-stimulated chemotaxis $=100$. Experiments performed with adult PMNs: filled squares $=\mathrm{CDCA}$; filled triangles $=$ UDCA. Experiments performed with neonatal PMNs: open squares $=$ CDCA; open triangles $=$ UDCA.

tissue concentrations of about $250 \mu \mathrm{M}$ are common in cholestasis (22).

Interestingly, only two bile acids - CDCA and its $\beta$-epimer UDCA - decrease, in a dose-dependent fashion, neutrophil recruitment, whereas both the more hydrophilic $\mathrm{CA}$ and the hydrophobic LTCA (23) were not effective. This inhibitory effect does not involve strong chemical bonds inasmuch as it is fully reverted by a simple washing procedure. Furthermore, the functional effect of UBAs appears unrelated to cytotoxicity. In fact, UDCA, although effective in regulating PMN locomotion, is significantly less toxic than the structurally similar CDCA
(Fig. 1). Intriguingly, functional impairment by CDCA and UDCA is fully reversed by co-incubation with both vitamins C and E, which may act as free radical scavengers. These data, taken together, demonstrate a specific UBA-cell interaction other than the detergent effect so often claimed to explain the many biologic effects of UBAs.

It has been previously demonstrated that high CDCA concentrations (100-200 $\mu \mathrm{M})$ cause irreversible cell damage using oxygen free radicals as intracellular messengers in rat hepatocytes $(18,24)$ and cultured macrophages $(25)$. Our data, though, show that substantially lower concentrations of both CDCA and UDCA are able to functionally impair in a reversible fashion the human PMN chemotaxis. Evidence from the experiments with antioxidant vitamins suggests that there is an intimate interplay between UBAs and free radicals in PMN locomotion, the mechanisms of which are currently being studied.

Our findings show that UDCA and CDCA are able to modulate the chemotaxis of both adult and neonatal PMNs. Thus, as neonates have a chemotactic motility significantly lower than the adults (14), the down-modulation induced by either UDCA or CDCA may further aggravate the natural impairment of neonatal PMNs.

In conclusion, we provide evidence that CDCA and UDCA modulate human PMNs chemotaxis in vitro. These observations may help explain both the complex immune derangement of the cholestatic patient and the immunomodulating potential of UDCA that has gained a wide therapeutic success.

Acknowledgments. The authors thank Dr. Ira H. Gewolb and Dr. Vincenzo Casolaro for critically reviewing the manuscript.

\section{REFERENCES}

1. Armstrong CP, Dixon JM, Taylor TV, Davies GC 1984 Surgical experience of deeply jaundiced patients with bile duct obstruction. Br J Surg 71:234-238

2. Holman JM, Rikkers LK, Moody FG 1979 Sepsis in the management of complicated biliary disorders. Am J Surg 138:809-813

3. Benkham JI, McPherson GAD, Blumgart LH 1984 Septic complications of percutaneous transhepatic biliary drainage. Am J Surg 147:318-321

4. Balistreri WF 1997 Bile acid therapy in pediatric hepatobiliary disease: the role of ursodeoxycholic acid. J Pediatr Gastroenterol Nutr 24:573-589

5. Combes B, Carithers Jr RL, Maddrey WC Munoz S Garcia-Tsao G, Bonner GF, Boyer JL, Luketic VA, Shiffman ML, Peters MG, White H, Zetterman RK, Risser R, Rossi SS, Hofmann 1999 Biliary bile acids in primary biliary cirrhosis: effect of ursodeoxycholic acid. Hepatology 29:1649-1654

6. Calmus Y, Poupon R 1991 Ursodeoxycholic acid (UDCA) in the treatment of chronic cholestatic diseases. Biochimie 73:1335-1338

7. Swain MG, Tjandra K, Kanwar S, Kubes P 1995 Neutrophil adhesion is impaired in a rat model of cholestasis. Gastroenterology 109:923-932

8. Tjandra T, Woodman RC, Swain M 1997 Impaired neutrophil microbicidal activity in rat cholestasis. Gastroenterology 112:1692-1698

9. Levy R, Schlaeffer F, Keynan A, Nagauker O, Yaari A, Sikuler E 1993 Increased neutrophil function induced by bile duct ligation in a rat model. Hepatology 17:908914

10. D’Odorico A, Melis A, Baragiotta A, Martines D, Naccarato R, Floreani A 1999 Oxygen-derived free radical production by peripheral blood neutrophils in chronic cholestatic liver disease. Hepatogastroenterology 46:1831-1835

11. Ohshio G, Miyachi Y, Kuso H, Niwa Y, Manabe T, Tobe T 1988 Effects of sera from patients with obstructive jaundice on the generation of oxygen intermediates by normal polymorphonuclear leukocytes. Liver 8:366-371

12. Helmut G 1976 Bile acids in hepato-biliary diseases. In: Nair P, Kritchevsky D (eds) The Bile Acids - Chemistry, Physiology and Metabolism. Vol. III. Plenum Press, New York, pp 59-60

13. Chen X, Yang D, Shen W, Dong HF, Wang JM, Oppenheim JJ, Howard OMZ 2000 Characterization of chenodeoxicolic acid as an endogenous antagonist of the Gcoupled formyl peptide receptors. Inflamm Res 49:744-755 
14. Hill HR 1987 Biochemical, structural and functional abnormalities of polymorphonuclear leukocytes in the neonate. Pediatr Res 22:375-382

15. Viggiano D, Romano G, Caniglia M, Santoro P, Palumbo A, Ciccimarra F 1994 Impaired LTB4 release by neonatal polymorphonuclear leukocytes. Pediatr Res 36:60-63

16. Santoro P, Agosti V, Viggiano D, Palumbo A, Sarno T, Ciccimarra F 1995 Impaired D-myo-inositol 1,4,5 triphosphate generation from cord blood polymorphonuclear leukocytes. Pediatr Res 38:564-567

17. Nilson E, Andersson T, Fällman M, Rosendahl K, Palmblad J 1992 Effects of ethanol on the chemotactic peptide-induced second messenger generation and superoxide production in polymorphonuclear leukocytes. J Infect Dis 166:854-860

18. Sokol RJ, Devereaux M, Khandwala R, O'Brien K 1993 Evidence for involvement of free radicals in bile acid toxicity to isolated rat hepatocytes. Hepatology 17:869-881

19. Sokol RJ, McKIm Jr IM, Goff MC, Ruyle SZ, Devereaux MW, Han D, Packer L, Everson G 1998 Vitamin E reduces oxidant injury to miochondria and the hepatotoxicity of taurochenodeoxicholic acid in the rat. Gastroenterology 114:164-174
20. Setchell KDR, Lawson AM Blackstock EJ, Murphy GM 1982 Diurnal changes in serum unconjugated bile acids in normal man. Gut 23:637-642

21. Lacaille F, Khazal P 1993 The immunosuppressive effect of ursodeoxycholic acid: a comparative in vitro study on human peripheral blood mononuclear cells. Hepatology 18:165-172

22. Greim H, Trülzsch D, Czygan P, Rudick J, Hutterer F, Schaffner F, Popper H 1972 Mechanism of cholestasis 6: bile acids in human livers with or without biliary obstruction. Gastroenterology 65:846-850

23. Roda A, Minutello A, Angellotti MA, Fini A 1990 Bile acid structure-activity relationship: evaluation of bile acid lipophilicity using 1-octanol/water partition coefficient and reverse phase HPLC. J Lipid Res 31:1433-1443

24. Krahenbuhl S, Talos C, Fisher S, Reichen J 1994 Toxicity of bile acids on the electron transport chain of isolated rat liver mitochondria. Hepatology 19:471-479

25. Ljubuncic P, Fuhrman B, Oiknine J, Bomzon A 1996 Effect of deoxycholic acid and ursodeoxycholic acid on lipid peroxidation in cultured macrophages. Gut $39: 475-478$ 